\title{
Pseudo-Cerebrospinal Fluid Rhinorrhea Resulting from Aberrant Cross-Innervation of Trigeminal and Facial Nerves following Skull Base Surgery
}

\author{
Benjamin L. Grannan, MD ${ }^{1}$ Wenya Linda Bi, MD, $\mathrm{PhD}^{1} \quad$ lan F. Dunn, MD ${ }^{1}$ \\ ${ }^{1}$ Department of Neurosurgery, Brigham and Women's Hospital, \\ Boston, United States \\ Address for correspondence lan F. Dunn, MD, Department of \\ Neurosurgery, Brigham and Women's Hospital, 15 Francis Street, \\ Boston, MA 02115, United States (e-mail: idunn@partners.org).
}

J Neurol Surg Rep 2015;76:e62-e64.

\author{
Abstract \\ Keywords \\ - nasal hypersecretion \\ - pseudo-cerebrospinal \\ fluid rhinorrhea \\ - trigeminal-facial \\ nerve regeneration
}

Objectives Pseudo-cerebrospinal fluid (CSF) rhinorrhea is a rare phenomenon, presumed to result from aberrant autonomic reinnervation of the paranasal mucosa following injury to the greater superficial petrosal nerve or periarterial plexus. In this report, we discuss the case of a postoperative patient with pseudo-CSF rhinorrhea exacerbated by chewing and propose a novel mechanism of pseudo-CSF rhinorrhea.

Case Report A 46-year-old man was found to have mastication-induced unilateral clear rhinorrhea following transtemporal resection of a cerebellopontine angle tumor. Computed tomography cisternogram and $\beta-2$ transferrin analysis proved negative for any evidence of CSF leak.

Conclusions Pseudo-CSF rhinorrhea exacerbated by mastication has not been previously reported in the literature. We propose aberrant cross-innervation of the trigeminal and facial nerves as the mechanism underlying the patient's condition. Pseudo-CSF rhinorrhea is an important entity to identify following skull base surgery because it may spare patients from the unnecessary invasive procedures and morbidity associated with the management of a presumed CSF leak.

\section{Introduction}

Cerebrospinal fluid (CSF) leak is a major complication of skull base surgery, occurring after $\sim 10 \%$ of transcranial skull base procedures, with ranges from $1 \%$ to $30 \%{ }^{1-4}$ Given the associated increased risk of central nervous system infection, prompt intervention often ensues following suspicion of a CSF leak. During management of CSF leak following skull base surgery, one should be aware of a rare and underappreciated entity known as pseudo-CSF rhinorrhea.

Pseudo-CSF rhinorrhea is a state of nasal mucosa hypersecretion resulting from autonomic dysregulation of the paranasal sinuses following skull base surgery or head trauma. ${ }^{5-7}$
This phenomenon was first described by Cusimano and Sekhar, who noted clear fluid hypersecretion without evidence of a dural CSF leak following skull base procedures involving exposure of the petrous or cavernous internal carotid artery and transection of the greater superficial petrosal nerve. $^{5}$ They hypothesized that the effect was due to injury to the periarterial sympathetic and parasympathetic fibers traveling to the nasal mucosa followed by aberrant reinnervation leading to a relative parasympathetic state. They also noted that the nasal hypersecretion tended to be exacerbated by physical activity, elevated ambient or body temperature, and intense emotional experience. Additionally, bilateral nasal hypersecretion, interpreted as pseudo-CSF received

January 24, 2014 accepted after revision

October 20, 2014

published online

January 16, 2015
DOI http://dx.doi.org/ 10.1055/s-0034-1396655. ISSN 2193-6366.

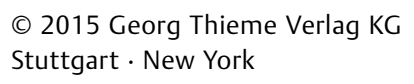

License terms

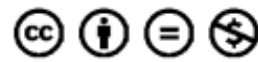


rhinorrhea, has also been described in the setting of traumatic skull fractures, possibly due to injury to parasympathetic nerves synapsing at the sphenopalatine ganglion, an area prone to trauma. $^{7}$

We present a case of pseudo-CSF fluid rhinorrhea in a patient who underwent a transtemporal craniotomy for resection of a cerebellopontine angle mass. The surgical intervention did not involve exposure of the petrous or cavernous segment of the internal carotid artery or injury to the greater superficial petrosal nerve, but it did involve dissection of a malignant invasive tumor from the facial and trigeminal nerves. Furthermore, the patient's nasal fluid hypersecretion was exacerbated by mastication rather than physical activity, elevated ambient or body temperature, or intense emotions. Given these distinct differences from previously described cases, we hypothesize a novel mechanism of trigeminal facial cross-innervation to underlie pseudo-CSF rhinorrhea in this patient.

\section{Case Presentation}

A 46-year-old man presented with 1 month of right-sided suboccipital headache and right facial numbness. Past medical history was negative for any malignancies or immunosuppressive conditions. Neurologic examination revealed decreased facial sensation, greatest in the ophthalmic distribution, partial in the maxillary distribution, and spared in the mandibular region of the trigeminal nerve, and mild rightsided facial weakness. Magnetic resonance imaging (MRI) revealed a $2.7 \times 1.9 \times 2.5 \mathrm{~cm}$ enhancing mass in the right cerebellopontine angle with significant surrounding edema and suggestion of dural enhancement entering the porus acusticus and Meckel cave ( - Fig. 1). Radiologic differential diagnosis included meningioma, vestibular schwannoma, extradural metastasis, or, less likely, a malignant glioma. A transtemporal craniotomy with partial mastoidectomy was performed, with resection of an intra-axial mass that coated the trigeminal and VII/VIII complex. Lymphoma was noted on the frozen diagnosis and confirmed on final pathology, with a decision to continue resection due to the significant amount of cerebellar edema. A watertight primary dural closure supplemented with fibrin glue and dural substitute was performed after buttressing the mastoid air cells with fat. The internal carotid artery and cavernous sinus were not exposed. Sensation in the maxillary distribution of the right trigeminal nerve and facial nerve function both improved postoperatively. Abnormal lacrimation was not noted.

On postoperative day 9, the patient noted clear fluid leakage from his right nostril that was initially exacerbated when moving into an upright position. A lumbar drain was placed for presumed CSF leak. However, computed tomography (CT) cisternogram failed to demonstrate any contrast opacification of the mastoid air cells, middle ear, or any other evidence of CSF leak. The nasal drainage abated after 2 days, and the lumbar drain was discontinued. The patient subsequently underwent seven cycles of chemotherapy, during which he experienced intermittent clear right nasal drainage, provoked by prolonged mastication, and ceased promptly

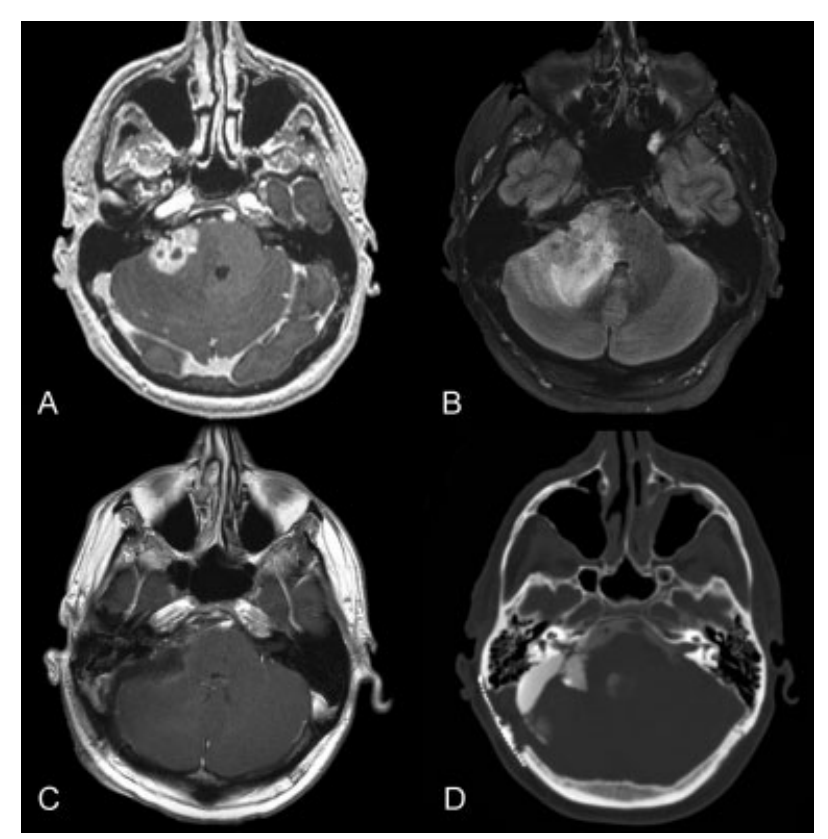

Fig. 1 (A) Axial T1-weighted gadolinium-enhanced magnetic resonance imaging (MRI) demonstrating a right cerebellopontine angle lesion at initial presentation, extending from the mid-pons to lower pontomedullary junction, with enhancement noted to extend to the internal auditory meatus. (B) Axial T2-weighted fluid-attenuated inversion recovery sequence demonstrating significant perilesional edema with partial effacement of the fourth ventricle. (C) Postoperative gadolinium-enhanced MRI with gross total resection of the tumor. (D) Computed tomography cisternogram for work-up of rightsided clear rhinorrhea failed to demonstrate any contrast opacification of the right mastoid air cells, right middle ear, or adjacent sinuses.

after finishing a meal. No association with position, activity, ambient temperature, emotion, or lacrimation was noted. A $\beta-2$ transferrin analysis of the nasal discharge was negative. The patient's facial nerve function was noted to be symmetrical without evidence of significant right facial palsy on follow-up evaluations. There was also no increased ipsilateral lacrimation. Multiple CT and MRI scans were negative for fluid collection in mastoid air cell, middle ear, eustachian tube, or paranasal sinuses. Sinusitis or any rhinologic infection was excluded, and there was no evidence of meningitis.

\section{Discussion}

Pseudo-CSF rhinorrhea, as originally described, is diagnosed based on the following criteria: recent skull base surgery involving petrous or cavernous internal carotid artery, rhinorrhea ipsilateral to procedure, diminished or absent ipsilateral lacrimation, no signs of meningitis, and negative $\beta-2$ transferrin testing of secreted fluid. ${ }^{5}$ It has been proposed to result from aberrant reinnervation of sympathetic or parasympathetic nerve fibers injured during skull base procedures with petrous or cavernous internal carotid artery exposure and sacrifice of the greater superficial petrosal nerve. Sympathetic innervation travels from the cervical sympathetic ganglia to the internal carotid artery to the oculomotor and trigeminal nerves to targets such as the 
paranasal sinus mucosa. Parasympathetic fibers travel from the superior salivatory nucleus to the nervus intermedius and facial nerve to the geniculate ganglion and greater superficial petrosal nerve before synapsing at the sphenopalatine ganglion to reach the paranasal mucosa. Interestingly, removal of either sympathetic input by stellate ganglion extirpation ${ }^{8}$ or parasympathetic input by sacrifice of the greater superficial petrosal nerve ${ }^{9}$ can result in nasal mucosa hypersecretion. Although abolition of sympathetic input logically leads to a relative parasympathetic state, it is not entirely clear why sectioning the greater superficial petrosal nerve would do the same. Hypotheses range from aberrant reinnervation leading to increased parasympathetic input, overcompensation of ancillary parasympathetic input, or hypersensitivity of postganglionic fibers arising from the sphenopalatine ganglion. 5,6

Nasal fluid secretion in the setting of pseudo-CSF rhinorrhea is classically exacerbated by physical activity during which relative hyperemia of the nasal mucosa is not properly countered by the vasoconstrictive effects of sympathetic input. Increased ambient or body temperature along with intense emotional experiences have also been associated with nasal hypersecretion. In our case, the patient noted that his nasal secretion became most noticeable during mastication and would promptly resolve after he stopped chewing. He also presented with a right facial nerve paralysis and trigeminal nerve sensory deficits in the maxillary and ophthalmic distribution, but there were no trigeminal motor deficits or atrophy of masseter or temporalis muscles. The connection between trigeminal activation of muscles of mastication and nasal fluid secretion suggests a trigeminal-facial cross-innervation, a phenomenon not previously reported in the scientific literature.

Such aberrant cross-innervation is often seen in the setting of synkinesis, or the involuntary motor innervation of a muscle upon activation of a nearby muscle. This most commonly presents after regeneration of the facial nerve following injury. Synkinesis involving two cranial nerves, although less common, has also been reported between the trigeminal and oculomotor or abducens nerves. ${ }^{10-12}$ Rubin et al reported facial-trigeminal synkinesis following resection of a trigeminal schwannoma through a transzygomatic temporal craniotomy, with resultant facial weakness and jaw deviation. ${ }^{13}$ Electrophysiologic stimulation of the facial nerve resulted in activation of the masseter and temporalis muscles, suggesting facial nerve rewiring to control muscles typically innervated by the trigeminal nerve. In contrast, the findings in our patient suggest that aberrant regeneration of the trigeminal and facial nerves may lead to trigeminal input on the autonomic nerve fibers of the facial nerve as demonstrated by the patient's increased vasomotor fluid secretion in response to chewing.

Our case suggests a novel presentation of facial-trigeminal cross-innervation leading to pseudo-CSF rhinorrhea in the absence of injury to sympathetic or parasympathetic nerve fibers, which are most commonly injured during operation involving petrous bone removal, sacrifice of the greater superficial petrosal nerve, exposure of the petrosal segment of the internal carotid artery, or invasion of the cavernous sinus. It is difficult to conclude whether this patient's trigem- inal-facial cross-innervation is the result of ephaptic transmission between damaged peripheral fibers or through central reorganization at the superior salivatory nucleus. Although synkinesis tends to be considered a result of peripheral regeneration, it has been shown to result from central lesions. ${ }^{14}$ Electrophysiology studies may help distinguish between the two for cases recalcitrant to repeat diagnostic work-up. Empirical treatments, such as atropine, may also be efficacious from a physiologic standpoint.

\section{Conclusion}

Pseudo-CSF rhinorrhea is a rare but clinically important differential diagnosis for unilateral nasal secretion following skull base surgery. Work-up and treatment of presumed CSF leaks presents significant morbidity for the skull base tumor patient population. The limited literature on this entity proposes an underlying dysautonomia due to a constant association with violation of the cavernous sinus and greater superficial petrosal nerve. The absence of these findings in our case in combination with the physiologic trigger of mastication prior to rhinorrhea suggests an alternative and novel mechanism of trigeminal and facial nerve cross-innervation. Early recognition and diagnosis of this important entity may spare patients from unnecessary invasive procedures.

\section{References}

1 Leonetti J, Anderson D, Marzo S, Moynihan G. Cerebrospinal fluid fistula after transtemporal skull base surgery. Otolaryngol Head Neck Surg 2001;124(5):511-514

2 Donald PJ. Complications in skull base surgery for malignancy. Laryngoscope 1999;109(12):1959-1966

3 Dos Santos LR, Cernea CR, Brandao LG, et al. Results and prognostic factors in skull base surgery. Am J Surg 1994;168(5):481-484

4 Selesnick SH, Liu JC, Jen A, Newman J. The incidence of cerebrospinal fluid leak after vestibular schwannoma surgery. Otol Neurotol 2004;25(3):387-393

5 Cusimano MD, Sekhar LN. Pseudo-cerebrospinal fluid rhinorrhea. J Neurosurg 1994;80(1):26-30

6 Hamada H, Endo S, Akai T, Ohi M, Kurimoto M, Takaku A. Pseudocerebrospinal fluid rhinorrhea after skull base surgery. Neurol Med Chir (Tokyo) 1998;38(6):371-373

7 Hilinski JM, Kim T, Harris JP. Posttraumatic pseudo-cerebrospinal fluid rhinorrhea. Otol Neurotol 2001;22(5):701-705

8 Fowler EP. Unilateral vasomotor rhinitis due to interference with the cervical sympathetic system. Arch Otolaryngol 1943;37:710-712

9 Golding-Wood PH. Observations on petrosal and vidian neurectomy in chronic vasomotor rhinitis. J Laryngol Otol 1961;75:232-247

10 McGovern ST, Crompton JL, Ingham PN. Trigemino-abducens synkinesis: an unusual case of aberrant regeneration. Aust N Z J Ophthalmol 1986;14(3):275-279

11 Shulman LM, Gallo BV, Weiner WJ. Acquired abducens-trigeminal synkinesis. Neurology 1998;50(5):1507-1508

12 Eve FR. Pterygoid-levator synkinesis. The Marcus Gunn jaw-winking phenomenon. J Clin Neuroophthalmol 1987;7(1):61-62

13 Rubin DI, Matsumoto JY, Suarez GA, Auger RG. Facial trigeminal synkinesis associated with a trigeminal schwannoma. Neurology 1999;53(3):635-637

14 Pullicino PM, Jacobs L, McCall WD Jr, Garvey M, Ostrow PT, Miller LL. Spontaneous palpebromandibular synkinesia: a localizing clinical sign. Ann Neurol 1994;35(2):222-228 